Marcia A. Docherty*

\title{
Sociocultural learning in emergency medicine: a holistic examination of competence
}

https://doi.org/10.1515/dx-2020-0001

Received January 5, 2020; accepted May 20, 2020; published online July 11, 2020

\begin{abstract}
Objectives: In the medical community of practice, the resident is situated in systems of professional and cultural activities. How diagnostic competence manifests in their sociocultural context is examined through situativity and systems theories. A holistic model is proposed that could examine diagnostic competence across micro (individual), meso (activity), and macro (cultural) systems.
\end{abstract}

Methods: Two short scenarios are presented resulting from observations of emergency medicine residents and their supervising physicians. These scenarios are analyzed using a trans-theoretical model of situativity and systems theories to understand how diagnostic competence manifests in practice (activity system).

Results: Assessment of diagnostic competence in a sociocultural context may require assessment of responses to contextual factors that seem immaterial to clinical reasoning. This information may signal that the resident also has the skills to identify appropriate information channels within an activity system and can accurately collect and prioritize clinical information within those channels. Therefore, the formal assessment of competent clinical reasoning performance, as a situated practice, may benefit from delineating how much of the context of an activity system is required to be competently managed and synthesized across the competency milestones.

Conclusions: The examination of diagnostic competence as a sociocultural practice can provide a unique and holistic examination of clinical reasoning performance and assessment.

Keywords: competence; communities of practice; emergency medicine; situativity theory; systems theory.

*Corresponding author: Marcia A. Docherty, Institute for Social Innovation, Fielding Graduate University, 2020 De la Vina Street, Santa Barbara, CA 93105-3814, USA,

E-mail: mdocherty@email.fielding.edu

\section{Introduction}

The recognition that clinical reasoning performance assessment can be situated in a contextual framework is an important shift in diagnostic competency assessment [1]. However, the complexity of contextual factors that impact the performance and assessment of clinical reasoning should not be underestimated. For example, the theories catalogued under situativity theory illustrate the complex ways situated cognition, distributed cognition, embodied cognition, and ecological psychology diverge yet also overlap [2]. Emerging evidence suggests that situativity theory could be useful in understanding how contextual factors impact clinical reasoning performance and a research agenda combining these broad theories alongside more precise theories is recommended [3]. In this paper, a trans-theoretical model of situativity and systems theories is combined to examine a novel way of understanding clinical reasoning performance in the complex context of medical practice. This model is then piloted on observational data of emergency medicine residents.

\section{Situated learning in the community of practice}

Situativity theory emerged from the seminal research of situated learning [4]. Situated learning describes the transition of the newcomer (novice) in a community of practice (CoP) to the old-timer (expert) through a process described as centripetal participation in the learning curriculum of the activity system, or legitimate peripheral participation [5]. A CoP is defined as "an activity system about which participants share understandings concerning what they are doing and what that means in their lives and for their communities" ([5], p. 98). A CoP can be as large as the entire field of medicine or faculty of medicine to as small as a local department within a hospital to the specialties within that department. Fundamental to the CoP, is the concept that mastery does not reside in the individual but is decentralized in the structuring structures of each unique CoP - for example, the tools that allow for sensory and motor input (embodied cognition), the affordances and effectivities (ecological psychology), the environmental 
interactions (situated cognition), how meaning is shared (distributed cognition), and so on [2]. This sociocultural approach also acknowledges that CoPs also contains cultural aspects such as culture, history, politics, economics, etc. which may cause the learner to learn things unintended or contradictory to the official learning agenda - such as learning to display knowledge for assessment purposes [5]. Situativity theory provides a useful framework for examining the individual as situated in systems of activity (practice) and culture. From this perspective, it is reasonable to wonder how can we legitimately assess individual clinical reasoning performance using situativity theory? Systems and complexity theories may hold the answer.

\section{Systems and complexity}

Situativity theory allows us to view medical knowledge and the development of clinical reasoning as a complex social phenomenon of practice and culture [6]. Systems and complexity theories (which overlap and diverge much like situativity theories) offer an explanatory framework of quality reasoning within individuals that could be applied to clinical reasoning. Systems contain a number of interdependent elements that can range from simple to complex and are largely self-organizing [7]. In complex systems, we are inundated with sensory information (feedback) and how we elect to filter that information in (attend to it) or filter it out (ignore it) determines the quality and competence of our resulting actions [7, 8]. For example, competent clinical reasoning requires not only identifying the clinical information that is key but also ignoring the clinical information that is not key. In ever-evolving systems, when we respond to information (or adapt), we trial an action (or solution) [9] which can range from wrong - less wrong - somewhat right - very right. In other words, the clinical information attended to impacts the accuracy of the diagnostic hypothesis. If the hypothesis is determined to be not accurate (error), alternative clinical information can then be considered (attended to) or not. Therefore, for competent clinical reasoning performance to occur in an ever-evolving system, one will always be adapting, trialing actions, and responding to error. The quality of adapting, trialing, and error (clinical reasoning performance) has been classified in the organizational sciences as single and double-loop learning [10] and generative learning [8]. Unfortunately, these classifications, like situated learning, do not distinguish between the activity and cultural systems.

A more complex cataloguing of adaptation was developed by Gregory Bateson [9] that may differentiate competent clinical reasoning between activity and cultural systems. Bateson's description of adapting, trialing and responding to error ranges from adaptation not subject to error (Zero Learning), adaptation between a set of alternatives (Learning I), adaptation that expands the set of alternatives (Learning II), adaptation that changes the set of alternatives (Learning III) [9]. It is important to note that these are not hierarchical classifications that one moves from lower levels to higher levels as one develops skill. Instead, these adaptations occur from decision to decision. Correlating this classification of systems thinking with situativity theory's concept of the individual situated in activity and cultural systems creates a novel, transtheoretical model of clinical reasoning performance. See Table 1.

As an individual, one must develop the foundational knowledge and skills of the profession that supports a minimally competent diagnosis - one learns the set of alternatives (Learning I or Knowledge Competence) [9]. The practice of clinical reasoning is developed first by obtaining declarative knowledge of a set of alternatives - common disease processes, standards, classic presentations, gold-standard management plans, etc. Learning theories such as cognitive, behavioral, constructivism [11] may be most appropriate for developing and assessing Knowledge Competence. Knowledge Competence alone is insufficient to reach entry-level competence in medicine and requires supervised practice within a CoP which contains both activity and cultural systems.

At the activity system level, the individual applies and transfers the profession's declarative knowledge through legitimate peripheral participation. This involves leveraging one's Knowledge Competence, collecting information from the system (such as the unique context of the patient, the availability of resources, the diverging opinions of peers, etc.) and reconciling one's knowledge with the situation at hand. The individual's Knowledge Competence expands into the activity system and the set of alternatives expands (Learning II or Situated Competence) [9]. This level of competence is challenging to achieve because of the numerous contextual factors that need to be filtered in and out and don't always travel through a complex system in a timely manner, a tendency to avoid mistakes, and a desire to reach autonomous practice as quickly as possible $[7-9,12]$. In an activity system, situativity [1-3], systems [7-9], and activity theories [13] may be most appropriate for supporting the development and assessment of Situated Competence.

Situated Competence also involves adapting practice within the activity system to the cultural norms of the CoP - such as its values, politics, economics, etc. [5, 6] These cultural norms are the habits, history and traditions (or 
Table 1: Clinical reasoning performance through a trans-theoretical model of situativity and systems theories. Systems theory describes how individuals make decisions in complex systems by adapting, trialing actions, and responding to error. Gregory Bateson [9] catalogued the increasing complexity of this adaptation, which when applied to situativity theory organizes competent clinical reasoning performance at an individual level (Knowledge Competence) and within a community of practice's activity (Situated Competence) and cultural (Cultural-Historical Competence) systems (see Figure 1).

\begin{tabular}{|c|c|}
\hline Bateson's categories of learning [9] & Clinical reasoning performance \\
\hline $\begin{array}{l}\text { Zero learning } \\
\text { - Minimal or no change in response to stimulus that is not } \\
\text { evaluative of correctness of response. } \\
\text { - Performance without consideration that the act may be in } \\
\text { error. }\end{array}$ & $\begin{array}{l}\text { Incompetence } \\
\text { - Insufficient medical knowledge. } \\
\text { - Presentation and management plan that is not subject to } \\
\text { correction. } \\
\text { - An incompetent and/or unethical diagnosis. }\end{array}$ \\
\hline $\begin{array}{l}\text { Learning I } \\
\text { - Change through conditioning that is limited to a set of un- } \\
\text { changing alternatives. } \\
\text { - Performance that allows for little choice and is limited in } \\
\text { context. }\end{array}$ & $\begin{array}{l}\text { Knowledge Competence } \\
\text { - Sufficient medical knowledge. } \\
\text { - Presentation and management plan that relies on classic } \\
\text { or gold-standard clinical findings. } \\
\text { - Unaware of the cultural-historical aspects of the commu- } \\
\text { nity of practice. } \\
\text { - Minimally competent diagnosis. }\end{array}$ \\
\hline $\begin{array}{l}\text { Learning II } \\
\text { - Corrective change that allows for alternative choices. } \\
\text { - Performance allows for an expanded range of choice } \\
\text { because the context is considered. }\end{array}$ & $\begin{array}{l}\text { Situated Competence } \\
\text { - Sufficient medical knowledge. } \\
\text { - Presentation and management plan that considers the } \\
\text { classic/gold-standard clinical findings situated in the } \\
\text { unique context of the patient, their family/caregivers, the } \\
\text { system. } \\
\text { - Decision making is influenced by the cultural aspects of the } \\
\text { community of practice. } \\
\text { - Highly competent diagnosis. }\end{array}$ \\
\hline $\begin{array}{l}\text { Learning III } \\
\text { - Corrective change of the standard alternatives presented as } \\
\text { choices. } \\
\text { - Performance that mindfully challenges the cultural norms } \\
\text { of practice. }\end{array}$ & $\begin{array}{l}\text { Cultural-Historical Competence } \\
\text { - Sufficient medical knowledge. } \\
\text { - Presentation and management plan that blends the } \\
\text { classic/ gold-standard clinical findings and the unique } \\
\text { context of the patient and system to determine innovative } \\
\text { and novel treatment plans. } \\
\text { - Questioning the habits, history and traditions of the com- } \\
\text { munity of practice. } \\
\text { - Competently transforming the practice. }\end{array}$ \\
\hline
\end{tabular}

Bateson did extend his classification into Learning IV but he did not describe it as he believed it did not currently occur in humans. Thus, Learning IV has been omitted here.

cultural-historical norms) of the CoP that structure the activity and knowledge systems. As an example, the Flexner Report structured medical practice in scientific methodologies [14]. Transformational practice change requires selecting a different set of alternatives (Learning III or Cultural-Historical Competence) [9]. Bateson did not believe that this level of competence is universally and regularly achievable [9] because we are subsumed by the practices of our $\mathrm{CoP}$ and think the set of alternatives can't be changed, lack the desire/power to change the set, or receive benefits from the status quo $[5,15]$. He posits that examining double binds (dilemmas, paradoxes) in the activity system can lead to the identification of rules, norms, practices that when challenged, can result in transforming the set of alternatives [16]. Unfortunately, this examination can also result in the manipulation of the activity system [16], which is beyond the scope of this paper. Culturalhistorical [6, 15], discourse and critical theories [17, 18] may be useful for the development and assessment of CulturalHistorical Competence.

\section{A holistic model of competence}

This trans-theoretical application of situativity and systems theories unifies competence across the individual 
knowledge system (micro), activity system (meso) and cultural system (macro) levels. See Figure 1. Competent performance varies within each of these systems (see Table 1). These micro-meso-macro systems are dynamic, which means competence is also a dynamic process. The newcomer (individual knowledge system) expands into the meso and macro systems until reaching full participation in the CoP. The idea of reaching full participation is misleading because all these systems continuously change and evolve. Perhaps a better metaphor is maintaining homeostasis, or optimal functioning. This model is piloted against observational data collected on fourth-year emergency medicine residents to determine whether it is useful for examining clinical reasoning performance and assessment.

\section{Methods}

Observational data presented here are excerpts from a larger case study collected in the emergency department of an academic, inner-city hospital located in a major Canadian city [19]. Two short scenarios are identified and qualitatively analyzed for examples of competence in the activity system (see Figure 1). The goal of this analysis is to pilot how Situated Competence manifests in the sociocultural system (see Table 1).

The observations were performed by the researcher who is not a physician, but has over 30 years of experience in the health care field as an allied health practitioner and educator. When examining the social worlds of others, the outsider perspective is helpful because it illuminates subsumed social practices [20].

Ethics approval for the original study was received from Fielding Graduate University (IRB No. 15-0603), the participating hospital's
Research Ethics Board, and participants gave informed consent before taking part.

\section{Results}

\section{Scenario one: arriving at a justifiable diagnosis}

A fourth-year resident and his supervising physician sit down at the physician's desk with 3 computers. The resident starts to present the case in bed \#1 (an older female with a rash) while the supervising physician enters information on the Emergency Department Information System (EDIS) computer. A senior medical student, stands behind the two. The resident searches the disease process he is considering on the Internet while the supervising physician brings up the patient's chest $x$-ray on the PACS system. Both the resident and the supervising physician closely examine the lower edges of the lungs. As the supervising physician pulls up the patient's lab results, paramedics walk by the desk with a patient lying calmly on a stretcher which catches the attention of the senior medical student but not the resident or the supervising physician. As the paramedic walks past the desk, the senior medical student returns her attention to the case at hand and inquires as to whether the patient had an ECG. The resident picks up the patient's chart to look while the supervising physician reviews the patient's history on the computer. The supervising physician discovers a history of psoriasis and

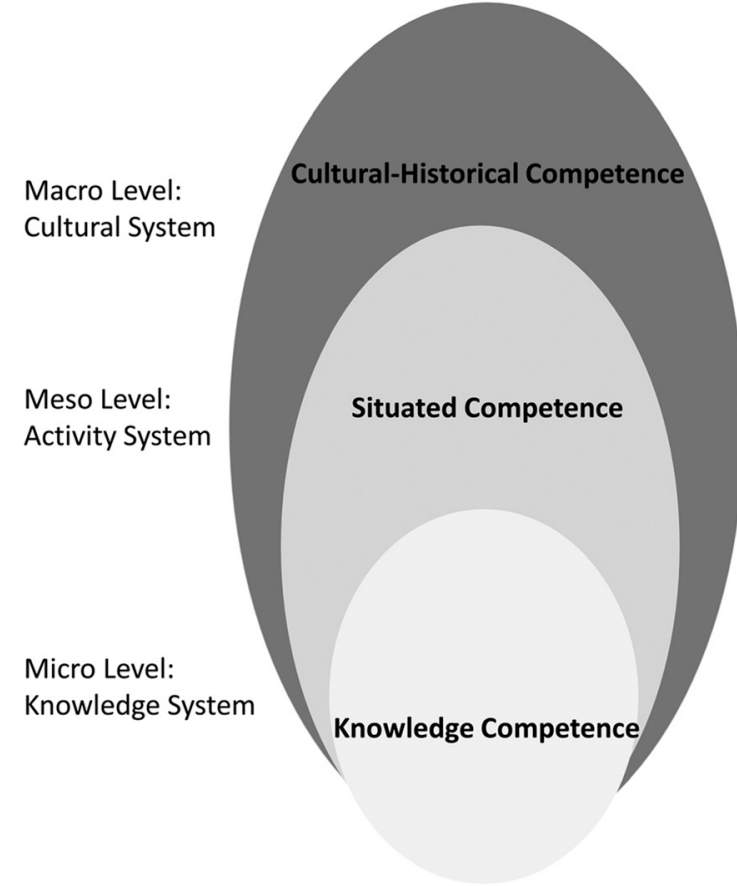

Corresponding Perspectives: Cultural-historical, discourse, critical theories $[6,15,17,18]$

Corresponding Perspectives: Situativity, systems, activity theories [1-3,7-9,13]

Corresponding Perspectives: Cognitive, behavioral, constructivist learning theories [11]
Figure 1: A holistic model of competence [19]. A trans-theoretical application of situativity and systems theories unifies competence across the individual knowledge system (micro), activity system (meso), and cultural system (macro) levels. From this perspective, competence manifests uniquely at each level (see Table 1) and requires corresponding theoretical perspectives for developing and assessing competence holistically. 
asks the resident for the chart. While the supervising physician reviews the admission history on the chart, the resident tells the senior medical student that her thought about an ECG is good. The supervising physician identifies relevant information in the admission history and shares it with the resident. He has decided to prescribe prednisone but is concerned it might interfere with further testing. They run through the details of the case again when the paramedic, who has transferred the incoming patient onto a bed, steps up to the desk with documentation. The senior medical student turns to get the documents while the supervising physician suggests to the resident that a dermatologist be called in. He then explains how to select one from the on-call list. They once again go through the patient's history, confirming the name and completing electronic documentation. As they start the next case, a nurse interrupts them to provide the status and vitals of the patient brought in by paramedics. The resident and senior medical student get up to see the new patient and the supervising physician begins dictating.

This scenario illustrates the application of key individual competencies to improve diagnostic accuracy and safety [21]. The senior resident has quickly formulated a competent problem representation and alongside the supervising physician, they focus on collecting the key clinical findings across multiple information channels, prioritize a diagnosis and treatment plan and confirm their findings twice before initiating the plan. This interaction, with the supervising physician taking the lead, illustrates competence in identifying the key clinical findings and prioritizing a diagnosis and treatment plan [21].

The observable activities supporting these competencies are filtering in and out information. Clinical findings are located across many information channels (the patient herself, the resident's physical examination, nursing documentation, electronic and paper-based diagnostic results) and with each case, a competent physician must determine which information channels to review (filter in) and which to ignore (filter out). There may also be a self-assessment on whether one is competent to collect information from a particular information channel. Then within each selected information channel, a competent physician must determine which clinical findings are key (filter in) and which are not (filter out). After this observation, the supervising physician explains that he uses (filters in) pattern recognition to improve his efficiency - the rash would influence (filter in and out) which information channels to review and what type of clinical findings might be key. This activity is an example of Situated Competence because they had sufficient medical knowledge and considered the unique context of the patient and the system.
During this process of filtering in and out clinical information to construct a diagnosis and treatment plan, the group was also observed filtering in and out information from the environment. The senior resident and supervising physician demonstrate this skill but the senior medical student, with much less clinical experience, did not. The supervising physician and resident filtered out the unnecessary information of the paramedic's activities and the senior medical student did not. The resident filtered in his supervision activities of the senior medical student while the supervising physician filtered that exchange out. All filtered in the arrival of the nurse at the desk. While these non-clinical filtering activities may not seem directly related to clinical reasoning performance, it illustrates the range of information circulating in a system. Assessing the novice's ability to maintain focus, switch focus, and return to previous topics when interrupted may indicate a level of Situated Competence that correlates to competent clinical reasoning performance, or at least a global assessment of readiness for entry-level practice.

\section{Scenario two: creating a shared mental model of a patient's illness}

A fourth-year emergency medicine resident has been assigned a male patient in the psychiatric observation room who was brought in by the police for angrily wielding a hammer in public. After interviewing the patient and reviewing his chart, the resident arranges a psychiatric consult. The following was observed:

Two psychiatric consulting physicians arrive. They are dressed in business attire while the resident is dressed in green scrubs. They huddle around the chart in the center of the nurses station. The resident is holding the patient's chart so that they can all read and discuss it together. The nurse from psychiatric emergency services joins the discussion. The resident highlights a family physician report that the patient is schizophrenic, doesn't take his medications, and is manic. They all review the chart again. The resident begins to outline her treatment plan when the nurse and one of the consulting physicians question the treatment plan and the validity of the family physician's report. "I have a bad feeling" is heard from the huddle, which causes the resident's supervising physician, who is working across from the nurses station, to look over at the group. The psychiatrists insist on a medical work up. The resident listens to them, agrees to the medical work up, and returns to her work. The psychiatric consultants continue to huddle and the nurse is complaining loudly about the family physician's report. The supervising physician calls the resident over to explain the situation and the supervising 
physician reviews the patient's admissions history. Then they both review his chart and confer with the patient's nurse until they are interrupted by a patient and return to other work. When I ask the resident what happened, she shrugs it off as a conflict over changing practice guidelines. She explains that a medical workup used to be required before requesting a psychiatric consultation but not under the new guidelines. She also admits that she might have also been in too much of a hurry to discharge the patient because he was difficult.

This scenario illustrates the application of key individual and team-based competencies to improve diagnostic accuracy and safety [21]. The resident examines the patient and reviews the chart in order to formulate a diagnostic hypothesis and treatment plan. She then initiates the treatment plan according to new practice guidelines and orders a psychiatric consultation. During the interprofessional consult, she shares her treatment plan and allows the group to review the chart, provide input, and modify her treatment plan. During this interaction, the resident could be assessed through observation as meeting the individual and team-based competencies for diagnosis [21].

Alternatively, this scenario can also illustrate how the resident attended to (filtered in) the new practice guidelines and the family physician's report and trialed her diagnostic hypothesis and treatment plan during the consult only to learn that psychiatric consultants filtered this information out. The resulting discord (error) resulted in loud voices that continue after the resident returns to her work. The supervising physician filters in this error and investigates by reviewing the patient's chart with the resident. Questioning the resident could confirm whether the resident applied competent clinical reasoning when formulating the treatment plan such as following the new practice guidelines (Knowledge Competence). The chart review by the supervising physician could be assessing whether the resident filtered in the right key clinical findings and did not filter out other relevant clinical findings (Situated Competence). Both of these activities are examples of how a supervising physician can assess clinical reasoning performance in context and highlight the importance of error for triggering that assessment.

\section{Discussion}

The individual competencies for diagnosis list six actions that are required for competent clinical reasoning [21]. There is little argument that a competent physician must have sufficient knowledge of clinical findings in order to determine if they are key or not (Knowledge Competence). These two scenarios suggest that equally important is the ability to effectively expand that knowledge by attending to information (feedback) from the patient and the system (Situated Competence). While this analysis does not examine assessment approaches for clinical reasoning performance, it does examine the observable features of competent clinical reasoning performance in the activity system (Situated Competence) that could be assessed.

In the first scenario, the resident is demonstrating a higher level of entrainment with the activity and cultural systems than the senior medical student. He is modeling and complementing the supervising physician activities, keeping focus on co-creating a justifiable diagnosis and treatment plan, and attending appropriately to his student (giving her encouragement) and the nurse (going to examine the new patient). In the second scenario, the resident is attending to contextual information and trialing a response (adapting). When the trial is not successful (error), she trials a new response.

Therefore, assessment strategies for Situated Competence may wish to include how well a resident adapts to a changing environment, how easily they maintain and change focus, how effective they are at filtering in and out background noise, how many activities they can manage concurrently, and how they respond to error. In other words, how much of the context of the activity system can they competently manage and synthesize. This information may signal that the resident also has the skills to identify appropriate information channels within an activity system and can accurately collect and prioritize clinical information within those channels. Therefore, the formal assessment of competent clinical reasoning performance, as a situated practice, may benefit from delineating how much of the context of an activity system is required to be competently managed and synthesized across the competency milestones [21]. For example, the advanced beginner milestone requires the competent use of 5 decision support tools and at the competent milestone, 10 decision support tools.

Effective assessment strategies also consider the context of the assessment [1]. In the first scenario, the supervising physician verbally checks the case details three times with the resident before finalizing a treatment plan. In the second scenario, the supervising physician checks the case details when conflict is overheard. This check involves questioning the resident and then reviewing the case to determine if anything needs correction. Both in situ assessments are brief check-ins that involve verbal check lists, open-ended questioning, and chart reviews. Therefore, informal assessments for clinical reasoning performance 
may wish to include similar approaches - random chart reviews and regular check ins. This approach does not align with the formal assessment of competence of the resident but instead ensures sufficient oversight of the resident, or ensuring the system supports diagnostic competence.

Examining clinical reasoning performance through situativity and systems theories is a perspective that is fundamentally different from cognitive and behavioral approaches because it examines how sociocultural systems can shape the individual mind [1-3]. A significant gap exists in the research on how best to research and analyze this process. Research examining shadow assessments (the informal assessments used in practice) and hidden curriculum (the passing down of culture) may provide valuable insights and direction for future research [22-24]. The Holistic Model of Competence (Figure 1) proposed here may provide a useful framework for organizing and expanding the examination of clinical reasoning performance as a sociocultural practice and lead to the development of multi-perspective assessment approaches.

\section{Limitations}

The use of observational research methods illustrates the challenge of reconciling clinical reasoning performance (an internal process) with observable contextual factors. In order to minimize the Hawthorne Effect, the researcher is required to obtain unobtrusive observations. However, to understand how the resident and supervising physician are making their decisions and evaluate the level of diagnostic competence, the researcher needs to interrupt the work with questions. Ethnomethodology, in which observations are video recorded and then reviewed with the participants after the observation, may be a more useful approach to reconcile thoughts with behavior [25]. Alternatively, group analysis of the observations by members of the CoP might also generate adequate distinctions of the level of diagnostic competence in use.

\section{Conclusions}

The examination of diagnostic competence as a sociocultural practice is valuable because these perspectives recognize that individuals are situated in systems of practice and cultural activities. Therefore, sociocultural approaches can provide a unique and holistic examination of clinical reasoning performance and assessment that may better represent the complexity of diagnosis in practice.
Research funding: None declared.

Author contributions: All authors have accepted responsibility for the entire content of this manuscript and approved its submission.

Competing interests: Authors state no conflict of interest. Informed consent: Informed consent was obtained from all individuals included in this study.

Ethical approval: Ethics approval for the original study was received from Fielding Graduate University (IRB No. 150603), the participating hospital's Research Ethics Board.

\section{References}

1. Rencic J, Schuwirth L, Gruppen L, Durning S. Clinical reasoning performance assessment: using situated cognition theory as a conceptual framework. Diagnosis 2020. https://doi.org/10.1515/ dx-2019-0051 [Epub ahead of print].

2. Torre D, Durning S, Rencic J, Lang V, Holmboe E, Daniel M. Widening the lens on clinical reasoning and error from "in the head" to "out in the world". Diagnosis 2020. https://doi.org/10. 1515/dx-2019-0098 [Epub ahead of print].

3. Rencic J, Schuwirth L, Gruppen L, Durning S. A situated cognition model for clinical reasoning performance assessment: a narrative review. Diagnosis 2020. https://doi.org/10.1515/dx-2019-00106 [Epub ahead of print].

4. Durning SJ, Artino AJ. Situativity theory: a perspective on how participants and the environment can interact: AMEE Guide no. 52. Med Teach 2011;33:188-99.

5. Lave J, Wenger E. Situated learning: legitimate peripheral participation. Cambridge, UK: Cambridge University Press; 1991/ 2011.

6. Bleakley A. Blunting Occam's razor: aligning medical education with studies of complexity. J Eval Clin Pract 2010; 16:849-55.

7. Bennet $A, B$ ennet $D$. Organizational survival in the new world: the intelligent complex adaptive system. Burlington, MA: Alex Bennet and David Bennet/Elsevier Science; 2004.

8. O'Connor J, McDermott I. The art of systems thinking. San Francisco, CA: Thorsons; 1997.

9. Bateson $\mathrm{G}$. The logical categories of learning and communication. In: Bateson G, editor. Steps to an ecology of mind. Northvale, N): Jason Aronson Inc; 1972/1987:pp. 205-44.

10. Argyris C, Schon D. Theory in practice: increasing professional effectiveness. San Francisco CA: Jossey-Bass; 1974.

11. Driscoll MP. Psychology of learning for instruction. Boston, MA: Allyn \& Bacon; 2000.

12. Ericsson KA. Deliberate practice and acquisition of expert performance: a general overview. Acad Emerg Med 2008;15: 988-94.

13. Engeström Y. Learning by expanding. An activity-theoretical approach to developmental research. Helsinki: OrientaKonsultit; 1987.

14. Cooke M, Irby DM, Sullivan W, Ludmerer KM. American medical education 100 years after the Flexner report. N Engl J Med 2006; 355:1339-44. 
15. Bourdieu P. Outline of a theory of practice (R. Nice, Trans.). Cambridge, UK: Cambridge University Press; 1977/2013.

16. Bateson G. Double bind. In: Bateson G, editor. Steps to an ecology of mind. Northvale, NJ: Jason Aronson Inc; 1972/1987:pp. 199-204.

17. Flick U. An introduction to qualitative research. Thousand Oaks, CA: Sage; 2009.

18. Sprague J. Feminist methodologies for critical researchers. Walnut Creek, CA: AltaMira Press; 2005.

19. Docherty M. Situated competence: towards a holistic definition of physician competence [dissertation]. Santa Barbara: Fielding Graduate University; 2017.

20. Emerson RM, Fretz RI, Shaw LL. Writing ethnographic fieldnotes. Chicago, IL: The University of Chicago Press; 2011.

21. Olson A, Rencic J, Cosby K, Rusz D, Papa F, Croskerry P, et al. Competencies for improving diagnosis: an interprofessional framework for education and training in health care. Diagnosis 2019;6:335-41.

22. Castanelli DJ, Weller JM, Molloy E, Bearman M. Shadow systems in assessment: how supervisors make progress decisions in practice. Adv Health Sci Educ 2020;25:131-47.

23. Lehmann LS, Sulmasy LS, Desai S. Hidden curricula ethics, and professionalism: optimizing clinical learning environments in becoming and being a physician: a position paper of the American college of physicians. Ann Intern Med 2018;168: 506-15.

24. Mulder H, ter Braak E, Chen HC, ten Cate O. Addressing the hidden curriculum in the clinical workplace: a practical tool for trainees and faculty. Med Teacher 2018;41:36-43.

25. Garfinkel H. Studies in ethnomethodology. Malden, MA: PrenticeHall: $1967 / 2014$. 